oophorectomies were performed. There was 21 diagnosis of ovarian cancer ( 7 patients among BRCA1 carriers and 14 patients among BRCA2 carriers). The mean age at diagnosis was 48.9 (SD 18.1) and 54.4 (SD 10.)9 years for BRCA1 and BRCA2 respectively $(\mathrm{p}=0.475)$.

The most frequent histology was high-grade serous ovarian adenocarcinoma (19 cases), 1 endometrioid carcinoma and 1 serous tubal adenocarcinoma. At the time of diagnosis, the most frequent stage was IIIC (9 cases) followed by stage IV (5 cases).

Regarding the treatment received, 11 primary surgeries were performed, in which primary complete cytoreduction was obtained in 10 of the cases. The other 10 cases received neoadjuvant chemotherapy (NACT) and surgery was subsequently performed. The median follow-up was 89 (EQR 34.5183.5) months. During this follow-up 6 patients died

Conclusion* To be a BRCA mutation carrier is associated with an increased risk of ovarian cancer at earlier ages than the general population, with no significant differences between the two types of mutation. The most frequent histology was highgrade serous ovarian adenocarcinoma and the most frequent stage at diagnosis was IIIC.

\section{BRCA-MUTATION-ASSOCIATED FALLOPIAN TUBE CARCINOMA}

${ }^{1} V$ Benito*, ${ }^{2} \mathrm{C}$ Molo, ${ }^{3} \mathrm{P}$ Garcia, ${ }^{4} \mathrm{~A}$ Lubrano. ${ }^{1}$ Vithas Las Palmas, Gynecology Oncology, Las Palmas de Gran Canaria, Spain; ${ }^{2}$ Vithas Las Palmas, Gynecology and Obstetrics, Las Palmas de Gran Canaria, Spain; ${ }^{3}$ Eurofins Megalab Canarias, Pathology, Las Palmas de Gran Canaria, Spain; ${ }^{4}$ C.H.U. Insular-Materno Infantil, Gynecology and Obstetrics, Las Palmas de Gran Canaria, Spain

\subsection{6/ijgc-2021-ESG0.295}

Introduction/Background* Fallopian tube carcinoma is a very rare tumor, comprising less than $1 \%$ of all gynecologic cancers and found primarily in postmenopausal women. Women with fallopian tube carcinoma are reported to have a higher frequency of inherited BRCA mutations than those with ovarian carcinoma or primary peritoneal carcinoma, approximately $30 \%$ of women with fallopian tube cancer have a mutation in BRCA1 or BRCA2. Described risk factors for BRCA mutations in these patients, are younger age (under 60) and women with a family history of breast or ovarian cancer.

Methodology The patients' clinical, pathological, and imaging data are presented. Likewise, the most relevant surgical videos are also presented.

Result(s)* We describe the case of a 58-year-old nulliparous patient with a family history of ovarian cancer who underwent surgery for suspected ovarian cancer with carcinomatosis. Complete debulking surgery was achieved after pelvic peritonectomy, total hysterectomy, bilateral salpingo-oophorectomy, radical omentectomy, right diaphragmatic peritonectomy and exeresis of several paraaortic enlarged lymph nodes. Histological examination showed the presence of a high-grade serous fallopian tube cancer, FIGO stage IIIB, TNM pT3b N0 M0. Mutational analysis confirmed the presence of a deleterious mutation in BRCA-1. She underwent platinum-based chemotherapy and maintanence therapy with olaparib. Currently, 3 years after treatment, she is alive without evidence of disease.

Conclusion* Given the high frequency of BRCA mutation, all patients diagnosed with invasive fallopian tube cancer should be considered candidates for genetic testing. Likewise, patients with germline BRCA1 gene mutations in whom a prophylactic oophorectomy is performed, removal of fallopian tubes should be strongly considered.

\section{STEP BY STEP TOTAL OMENTECTOMY AND ANATOMICAL BOUNDARIES ON LIVE PATIENT (OPEN TECHNIQUE)}

I Selcuk*, HR Yalcin. Ankara Şehir Hastanesi, Turkey

\subsection{6/ijgc-2021-ESG0.296}

Introduction/Background* To demonstrate the proper anatomical boundaries and surgical technique for total omentectomy on a live patient.

Methodology This surgical video was recorded at Ankara City Hospital, an ESGO Accredited Gynecologic Oncology Center. Result(s)*

- The greater omentum attaches the stomach to the transverse colon. The double fold of the peritoneum encircling the stomach turns over itself and attaches to the transverse colon and transverse mesocolon. By the way, the greater omentum has four peritoneal folds.

- The greater omentum lies cranially from the greater curvature of the stomach and proximal duodenum, traverses over the transverse colon between the hepatic flexure at the right side and splenic flexure at the left side, and afterwards lies over the small intestines covering the whole abdominal cavity.

- Firstly, the posterior peritoneal fold covering the omentum which attaches to the transverse colon is cut, so an avascular plane is identified superior to the transverse mesocolon.

- Secondly, after cutting the posterior peritoneal fold of the omentum between the hepatic flexure and splenic flexure (the attachments at the level of hepatic and splenic flexure are dissected), the transverse colon is grasped caudally, and the omentum is grasped cranially. By the way, the gastrocolic space is easily dissected and developed. As a consequence, the omentum is dissected from the transverse mesocolon.

- The stomach and the transverse colon are separated, the omentum is found attached to the stomach, and the bursa omentalis is accessed. Afterwards, the omentum is excised from the hepatic flexure to the splenic flexure below the level of the greater curvature. If there is not any gross tumor nodule at this part, the gastro-epiploic vessels are preserved.

- While excising the omental structure between the stomach and spleen, care should be taken for short gastric vessels lying here, especially at the cranial part of this area. Besides, close to the splenic hilum, the omental tissue should be discriminated from the tail of the pancreas.

Conclusion* Developing the gastrocolic space is crucial while performing a total omentectomy. In addition, the peritoneal folds of the omentum should be dissected clearly and gently. 